\title{
Patrick Poch: Porträtgalerien auf Papier. Sammeln und Ordnen von druckgrafischen Porträts am Beispiel Kaiser Franz' I. von Österreich und anderer fürstlicher Sammler. Köln-Weimar-Wien: Böhlau 2018. ISBN: 978-3-205-20529-6.
}

\section{Open Access verfügbar unter: https://doi.org/10.7767/9783205208556}

Die vorliegende Publikation ist das Ergebnis eines vom Fonds zur Förderung der wissenschaftlichen Forschung (FWF) unterstützten Projektes. Patrick Poch liefert hier den zweiten Band der Reihe Geschichte der Familien-Fideikommissbibliothek des Hauses Habsburg-Lothringen. Die Gesamtleitung liegt bei Hans Petschar, dem Direktor des Bildarchivs und der Grafiksammlung der Österreichischen Nationalbibliothek Wien. Die Publikation Porträtgalerien auf Papier ist die Fortsetzung eines breit angelegten Forschungsvorhabens, dem bereits eine fachlich fundierte Publikation vom Autorentrio Huber-Frischeis, Knieling und Valenta zur Privatbibliothek Franz' I. vorausgegangen ist (2015) ${ }^{1}$. Mit der vorlie-

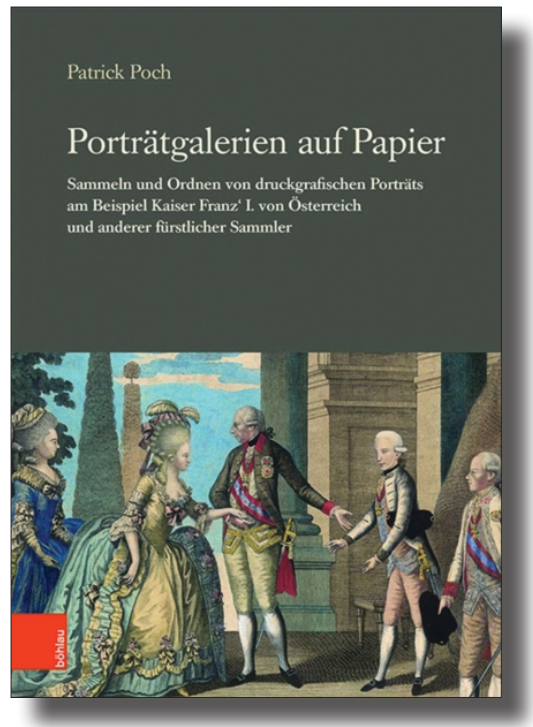
genden Arbeit wird erneut ein Schlaglicht auf die „verkannte“ Person Kaiser Franz II./I. geworfen. Ein wesentliches Ergebnis der beiden Publikationen ist der Nachweis, dass Franz neben Kaiser Rudolf II. und den Erzherzögen Ferdinand II. und Leopold Wilhelm ein weiterer - bislang nicht wahrgenommener und unterschätzter - bedeutender Sammler des Hauses Österreich gewesen zu sein scheint.

Patrick Poch legt bei seiner Untersuchung den Fokus auf den Bestand der druckgrafischen Porträts, die Kaiser Franz I. bereits als Erzherzog, dann als Oberhaupt des Heiligen Römischen Reiches und ab 1804/06 als erster österreichischer Kaiser ansammelte. Der Autor befasst sich aber nicht ausschließlich mit dieser speziellen Kollektion, sondern liefert zudem eine allgemeine Abhandlung über den Stellenwert der neuzeitlichen Porträtgrafik. Auch versucht er sich an einem Vergleich der habsburgi- 
schen mit anderen, zeitlich wie strukturell ähnlich gearteten, fürstlichen Porträtstichsammlungen. Das detaillierte Inhaltsverzeichnis gibt bereits Einblick in die klare und pointierte Vorgehensweise des Autors. Die Betrachtungen erfolgen dabei linear und zeitlich chronologisch.

Die Einleitung liefert zunächst eine Rückschau zu den Wurzeln des Sammelns, im speziellen von Bildnissen, bei Erzherzog Franz. Poch führt dabei richtig aus, dass das Interesse an Porträts auf der Ausbildung des Habsburgers in Florenz basierte, da Porträts als pädagogisches Hilfsmittel im Unterricht verwendet wurden. Auch die vom jugendlichen Erzherzog selbst gefertigten Zeichnungen zeugen vom frühen Interesse. Die Tätigkeit des Sammelns im Allgemeinen entsprach dabei seinem ordnenden Charakter. Es kann rekonstruiert werden, dass Franz historisch-genealogische Literatur und zeitgenössische Sammlungsratgeber (Apin 1726) zu Rate zog. Informationen über die Struktur des Sammelns und den Aufbau der Bildnissammlung, die Literatur- und Quellenlage zu diesem besonderen Bestand in der heutigen Österreichischen Nationalbibliothek sowie die aktuellen Strukturen (Aufstellung, Findbücher) vor Ort werden im Überblick gegeben. Das franzisceische Ordnungssystem basierte auf der ständischen Ordnung der Gesellschaft um 1800, orientierte sich aber auch an der Herkunft und den verschiedenen Berufsgruppen der Dargestellten. Unmittelbar nach dem Tod von Franz I. im Jahr 1835 wurde ein „Kassasturz“ der Sammlung vollzogen, bei dem 66.709 Bildnisse vorgefunden wurden.

Die der Einleitung folgenden beiden Kapitel der Publikation beleuchten chronologisch den Beginn des Sammelns bis 1785, reichen dann bis 1791 und markieren damit den Amtsantritt als römisch-deutscher Kaiser. Dieser stellte eine Zäsur für den Sammler und für seine Kollektion dar. Die Kaiserwürde beschränkte den Sammler im Zeitmanagement, ermöglichte ihm andererseits aber größere finanzielle Mittel und ein ausgereifteres System der Akquirierung. Poch weist auf die helfenden „Hintermänner“ hin, Thomas Young und Leopold von Khloyber, die entscheidenden Einfluss auf die Erweiterung der Sammlung und ihre Ordnung hatten. Beim Aspekt Bildniserwerb wird detailliert auf den damals üblichen Handel mit Grafiken, ihre Verfügbarkeit auf Messen und bei Versteigerungen hingewiesen. Als Mittelsmänner für den Monarchen waren neben Wiener Buchhändlern auch ausländische Kunsthändler tätig. Ebenso wurden österreichische Diplomaten an fremden Fürstenhöfen bemüht, nach Porträtstichen zu suchen. Eine bedeutende Erweiterung, nicht nur in der Stückzahl sondern auch in ihrer thematischen Ausrichtung und Systematik, erfuhr die kaiserliche Sammlung durch den Ankauf der Sammlung des Hannoveraners Georg Friedrich Brandes im Jahr 1796. Interessantes Detail ist der Umstand, dass die Bildnislei- 
denschaft von Kaiser Franz in Künstlerkreisen wohl bekannt war, weshalb zeitgenössische Künstler mit unterschiedlichem Erfolg immer wieder den Versuch unternahmen, ihre Werke dem Hof anzubieten.

Ein weiteres Kapitel widmet sich dem Stellenwert und der Bedeutung der Porträtgrafik im 18. Jahrhundert. Für diesen Exkurs werden bedeutende bürgerliche Sammlungen in Deutschen Landen und in der Habsburgermonarchie aufgelistet. Klar erkennt man hier den erziehenden und bildenden Charakter, den man Porträts beimaß, beeinflusst von den sog. viri illustri-Reihen der Antike, die im Zuge des Renaissance-Humanismus ihre Rezeption erfuhren. Poch widmet sich auch dem Stellenwert der Bildnisgrafik in fürstlichen Kunstkammern der frühen Neuzeit und zeichnet anhand der Sammlungen König Ludwigs XIV. von Frankreich und des sächsischen Kurfürsten und polnischen Königs August des Starken den Wandel hin zum spezialisierten Grafikkabinett des 17. und 18. Jahrhunderts nach.

Mängel lassen sich im Kapitel über die Kunstkammern feststellen. Kritisch ist hier die teilweise als veraltet einzustufende herangezogene Literatur zu sehen. Unerwähnt bleiben etwa im Falle der Dresdner Kunstkammer die 2010 herausgegebenen Inventarpublikationen sowie ein Band zur Sammlungsgeschichte. ${ }^{2}$ Ohne Belege wird etwa die Gründung der Ambraser Sammlung mit 1576 sowie jene Rudolfs II. in Prag mit „um 1590“ ausgewiesen. Im Ambraser Falle etwa gibt es kein eindeutiges Datum. Als Richtlinie dient das Jahr 1572, mit der Fertigstellung des Sammlungsgebäudes (Unterschloss). Auch die zu den Ambraser Sammlungen rezente Literatur (Ausstellungskataloge und Publikationen zu habsburgischen Kunstkammern) werden nicht miteinbezogen. So hätte die falsche Interpretation der Ambraser Rüstkammern, die nicht nur aus der erwähnten „Heldenrüstkammer" bestand, sondern wesentlich umfangreicher waren, vermieden werden können. Ebenso ist die Annahme, dass „die ursprüngliche Anordnung [...] nur mehr vage aus einem Inventar [...] von 1788“ nachvollziehbar ist, unrichtig, da allein für das 17. Jahrhundert sechs informative Ambraser Sammlungsinventare existieren.

Ein umfangreiches Kapitel widmet sich dem Sammeln und Ordnen druckgrafischer Porträts fürstlicher Sammlungen, die zeitlich wie strukturell mit jener Franz' II./l. zu vergleichen ist. Der Autor vergleicht dabei die Kollektionen des Prinzen Eugen von Savoyen, jene des bayerischen Kurfürsten Carl Theodor sowie die des „Bürgerkönigs“ Louis Philippe von Frankreich. Besonders ausführlich wird dabei die wechselvolle Geschichte der Porträtstichsammlung des Prinzen Eugen behandelt. Diese wurde vom Kaiserhaus angekauft, dem Bestand der kaiserlichen Hofbibliothek zugeordnet und 1845, ohne deren Ordnungsstruktur aufzuheben, der Kupferstichsammlung einverleibt. 
Trotz des Informationsgehaltes erhebt sich die Frage, warum der Exkurs zur Porträtgalerie im Musée historique in Schloss Versailles, wo ja bekanntlich nur Gemälde und keinerlei Stiche verwahrt werden, gemacht wird. Ebenso wirkt der wohl zu ausführliche Abriss über die Wiener Bevölkerungsstruktur zur Zeit Franz' I. wie ein Fremdkörper in der Publikation. Die Publikation schließt mit einem Kapitel zur Ordnung innerhalb der Porträtstichsammlung bei Franz I. Es wird dabei die Systematik aufgezeigt, die oft vom Kaiser eigenhändig erarbeiteten Hand- und Findbücher vorgestellt sowie auf die diversen Bestandskataloge verwiesen, die während der unterschiedlichen Sammlungsphasen entstanden sind.

Im knappen Resümée darf bezweifelt werden, ob Kaiser Franz I. seine Porträtstichsammlung tatsächlich aus Gründen der fürstlichen Repräsentation zusammentrug, wie dies durchaus bei anderen fürstlichen und adeligen Sammlern der Fall gewesen sein mag. Bei Franz spricht u.a. dagegen, dass er seine Sammlung als Privatangelegenheit, als Ergänzung seiner persönlichen Bibliothek ansah und diese wohl nur ausgewählten Besuchern präsentiert worden ist.

Den Abschluss der Abhandlung bildet ein gut strukturiertes und umfangreiches Abbildungs- Quellen- und Literaturverzeichnis sowie ein Personenregister. Informativ und wichtig sind die im Anhang angeführten Tabellen mit Ordnungsklassen zu den Sammlungen von Prinz Eugen, zu jener König Louis Philippes und schließlich zu der von Franz I.

Positiv zu bewerten sind sowohl die verständliche Sprache, die flüssige Schreibweise sowie der ausreichende Anmerkungsapparat. Das Layout ist gefällig und kann anhand zahlreicher Abbildungen, deren Größe gleichzeitig ihre Wertigkeit ausdrückt, als gelungen bezeichnet werden. Patrick Poch ist für die vorliegende Arbeit zu druckgrafischen Porträts im Allgemeinen, zur Bildnissammlung Kaiser Franz' I. von Österreich im Speziellen, zu gratulieren. Im Anmerkungsapparat ist auch die breite und fachliche Vernetzung des Autors erkennbar. Gespannt darf man nun auf den letzten Band aus der Reihe Geschichte der Familien-Fideikommissbibliothek des Hauses Habsburg-Lothringen sein, die der Geschichte der Fideikommissbibliothek von 1835 bis 1921 gewidmet sein wird.

Dr. Thomas Kuster

Kunsthistorisches Museum Wien, Schloss Ambras Innsbruck E-Mail: thomas.kuster@schlossambras-innsbruck.at 
1 Thomas Huber-Frischeis, Nina Knieling, Rainer Valenta (Hgg.), Die Privatbibliothek Kaiser Franz'I. von Österreich. 1784-1835. Bibliotheksund Kulturgeschichte einer fürstlichen Sammlung zwischen Aufklärung und Vormärz, Wien 2015.

2 Dirk Syndram, Martina Minning (Hgg.), Die Inventare der kurfürstlichsächsischen Kunstkammer in Dresden, 4 Bde., Dresden 2010; Dies. (Hgg.), Die kurfürstlich-sächsische Kunstkammer in Dresden. Geschichte einer Sammlung, Dresden 2012.

DOI: https://doi.org/10.31263/voebm.v72i1.2287

(C) Thomas Kuster

Dieses Werk ist lizenziert unter einer

Creative-Commons-Lizenz Namensnennung 4.0 International 